\title{
O Efeito da Terapia Comportamental para Enurese sobre Outros Problemas de Comportamento ${ }^{1}$
}

\author{
Rodrigo Fernando Pereira ${ }^{2}$ \\ Noel José Dias da Costa \\ Marina Monzani da Rocha \\ Mariana Castro Arantes \\ Edwiges Ferreira de Mattos Silvares \\ Universidade de São Paulo
}

\begin{abstract}
RESUMO - O objetivo do estudo foi investigar o efeito do tratamento para enurese sobre os escores de outros problemas de comportamento. Foram coletadas as informações de 97 prontuários de crianças e adolescentes atendidos no período de 2002 a 2006 em uma clínica-escola de psicologia, em programa específico para enurese com uso do alarme de urina. Os dados sobre problemas de comportamento foram avaliados por meio do Child Behavior Checklist, respondido pelas mães antes e depois do tratamento. Foi encontrada uma redução significativa nos escores de problemas de comportamento, independentemente do sucesso ou não no tratamento para enurese.
\end{abstract}

Palavras-chave: enurese; problemas de comportamento; terapia comportamental; Child Behavior Checklist.

\section{The Effect of the Behavioral Treatment for Enuresis on Other Behavior Problems}

\begin{abstract}
This work aimed to investigate the treatment effect for enuresis on other behavior problems' scores. Clinical records of 97 enuretic children and adolescents, receiving urine alarm treatment in a university clinic center from 2002 to 2006, were analyzed. Behavior problems' data were assessed through the Child Behavior Checklist, filled by the mothers at the beginning and at the end of the treatment. Results indicate a significant reduction on behavior problems' scores at the end of treatment, despite of enuresis treatment outcome.
\end{abstract}

Keywords: enuresis; behavior problems; behavior therapy; Child Behavior Checklist.

É recorrente a constatação de que a enurese apresenta-se com frequência associada a outros problemas de comportamento. De acordo com uma revisão recente, observa-se, em praticamente todos os estudos sobre comorbidade, uma maior probabilidade de as crianças enuréticas apresentarem problemas de comportamento, transtorno de hiperatividade e déficit de atenção (Baeyens, Roeyers, Vande Walle \& Hoebeke, 2005).

Um estudo brasileiro comparou o perfil comportamental de crianças enuréticas e crianças encaminhadas para atendimento psicológico em cinco clínicas-escolas, a partir de questionários respondidos pelos pais (Santos \& Silvares, 2006). Foi encontrada uma associação entre enurese e problemas de comportamento internalizantes, mas, de um modo geral, os pais de crianças enuréticas indicaram menos problemas do que os pais de crianças encaminhadas para assistência psicológica devido a outras queixas.

Diversos pontos ainda precisam ser esclarecidos na correlação entre enurese e problemas de comportamento. Tal

1 Financiamento: FAPESP e CNPq.

2 Endereço para correspondência: Av. Prof. Mello Moraes, 1721, Bloco F - Sala 30. Cidade Universitária. São Paulo, SP. CEP 05508-030. Tel.: (11) 3091-4173. E-mail: rpereira@usp.br. relação poderia ser dependente de fatores de risco comuns, como é sugerido por um estudo que apontou baixo nível socioeconômico como fator subjacente à relação entre problemas de comportamento e enurese (Van Hoecke, Baeyens, Vande Walle, Hoebeke \& Roeyers, 2003).

Outra hipótese seria a existência de uma relação causal entre enurese e problemas de comportamento. Não há evidências de que problemas de comportamento levem à dificuldade do controle urinário durante a noite. Por outro lado, existem estudos que apontam melhoras nos problemas de comportamento após o tratamento para enurese, sugerindo que problemas de comportamento são mais consequência do que causa da enurese. Longstaffe, Moffatt e Whalen (2000) encontraram reduções significativas nos escores de problemas de comportamento após seis meses de tratamento para enurese, independentemente do resultado e do tipo oferecido [placebo, Acetato de Desmopressina (DDAVP) e alarme]. Hirasing, Van Leerdan, Bolk-Bennik e Koot (2002) estudaram os efeitos de um tratamento para enurese com alarme de urina sobre outros problemas de comportamento e verificaram que $58 \%$ das crianças que apresentavam escores clínicos e limítrofes de problemas de comportamento passaram a apresentar escores na faixa normal após tratamento bem sucedido. 
A eficiência do tratamento da enurese com uso de alarme de urina tem sido objeto de várias pesquisas nos últimos anos. Algumas procuraram investigar os efeitos dos problemas de comportamento sobre os resultados desse tipo de tratamento, mas os dados acumulados são conflitantes.

Há estudos em que o tratamento com o alarme foi igualmente efetivo para crianças enuréticas com ou sem outros problemas de comportamento (Hirasing \& cols, 2002; Sacks \& De Leon, 1978). Em outros, entretanto, as desistências e fracassos do tratamento com alarme para enurese foram mais prováveis quando as crianças apresentavam problemas de comportamento associados (Arantes, 2007; Devlin \& O'Cathain, 1990; Ehrlick, Mellon, Whiteside, Witts \& Choma, 2005; Geffken, Johnson \& Walker,1986; Moffatt \& Cheang; 1995; Wagner \& Johnson, 1988).

A eficácia da psicoterapia comportamental para enurese com uso do alarme de urina vem sendo investigada em linha de pesquisa desenvolvida desde 1992 no Laboratório de Terapia Comportamental da Universidade de São Paulo (Projeto Enurese) (Costa, 2005; Pereira, 2006; Silva, 2004; Silvares, 2002). Tendo em vista as questões abertas quanto à associação entre enurese e problemas de comportamento, já foram realizados alguns trabalhos no Projeto Enurese (Arantes, 2007; Santos \& Silvares, 2006), mas ainda nenhum com o foco nos efeitos do tratamento para enurese sobre outros problemas de comportamento.

Assim, justifica-se a necessidade de realização do presente estudo, que teve como objetivo verificar se havia variação nos problemas de comportamento identificados em crianças e adolescentes atendidos pelo Projeto Enurese, quando comparados seus escores antes e depois do tratamento. Analisouse, ainda, se as variações observadas estavam associadas ao sucesso do tratamento.

\section{Método}

\section{Participantes}

Foram coletadas as informações de prontuários clínicos de 97 enuréticos, com idades entre 6 e 18 anos, atendidos pelo Projeto Enurese entre 2002 e 2006 . Foram excluídas as crianças cujas famílias desistiram do atendimento antes do seu término programado e aquelas em cujos prontuários não constavam dados do instrumento de medida utilizado, geralmente em função da dificuldade de contato com os pais.

A partir dos critérios de exclusão, restaram 61 participantes com idades entre 6 a 18 anos, sendo 44 do sexo masculino e 17 do sexo feminino. Dentre os participantes, 52 atingiram o critério de sucesso do tratamento, que consistia em permanecer 14 noites consecutivas sem episódios de enurese.

Os atendimentos realizados pelo Projeto Enurese envolvem diversos projetos, que foram aprovados pelos Comitês de Ética em Pesquisa do Instituto de Psicologia da Universidade de São Paulo e da Universidade Federal de São Paulo.

\section{Instrumento}

O instrumento utilizado para avaliação dos problemas de comportamento foi o Child Behavior Checklist-CBCL (Achenbach, 1991). Desenvolvido nos Estados Unidos, nos anos 70, busca medir a competência social (CS) e problemas comportamentais das crianças e adolescentes de 4 a 18 anos, a partir do ponto de vista dos pais ou responsáveis.

O CBCL é originalmente composto por 138 itens, 20 direcionados para avaliação das competências e 118 para avaliação de problemas de comportamento através de uma escala Likert de 3 pontos: 0 = não é verdadeira, tanto quanto sabe; 1 = um pouco verdadeira ou algumas vezes verdadeira; e 2 = muito verdadeira ou frequentemente verdadeira. A versão brasileira inclui todos os itens que compõem o questionário original, que foi elaborado utilizando a abordagem bottom-up (Achenbach \& Rescorla, 2007): ou seja, primeiro foi elaborada uma lista com diversos problemas de comportamento que crianças e jovens podem apresentar para, então, se encontrar as escalas de problemas de comportamento empiricamente baseadas, a partir da análise fatorial da lista original. Os nomes dados aos oito fatores encontrados foram: Isolamento, Queixas Somáticas, Ansiedade/Depressão, Problemas Sociais, Problemas de Pensamento, Problemas de Atenção, Comportamento Delinquente e Comportamento Agressivo (Achenbach, 1991). Esses fatores são, também, agrupados em: (a) Escala de Internalização (DI), que engloba as três primeiras escalas; (b) Escala de Externalização (DE), que engloba as duas últimas escalas; e (c) Escala Total de Problemas de Comportamento (DT), composta por todos os itens de problemas de comportamento do questionário.

No Brasil, foi realizado um estudo de validação preliminar do CBCL, utilizando a avaliação psiquiátrica como modelo de comparação dos resultados obtidos por meio desse inventário (Bordin, Mari \& Caeiro, 1995). A avaliação feita pela mãe, que respondeu ao CBCL, foi comparada com a avaliação feita por um psiquiatra, que não teve acesso ao inventário respondido pela mãe. Foi encontrado um alto índice de correlação entre as duas avaliações, o que sugere que o uso do questionário é válido no Brasil. No entanto, ainda não possuímos notas de corte brasileiras. Sendo assim, os valores-padrão adotados no Projeto Enurese para análise de nível clínico dos escores para as escalas DI, DE e DT são extraídos do estudo de normatização norte-americana (Achenbach, 1991): faixa clínica - escores iguais ou superiores a 60; faixa normal - escores inferiores a 60. A faixa clínica corresponde ao escore de $2 \%$ das crianças não encaminhadas para a clínica da amostra normativa americana (Achenbach, 1991).

\section{Procedimento}

Os dados dos participantes foram levantados a partir do banco de dados do Projeto Enurese. Foram analisados os valores dos escores obtidos nas escalas globais dos CBCLs preenchidos pelas mães antes e depois do tratamento. A partir desses dados, foi realizada uma análise estatística, com auxílio do SPSS for Window - release 10.01 standard 
version - SPSS Inc. e do Statistics for Window - release 4.2 - StatSoft Inc. STATA Statistics Data Analysis - release 5 Stata Corporation, utilizando teste de médias para verificação da variação entre as duas medidas (antes e depois do tratamento); teste de McNemar para identificação da ocorrência de mudanças entre as faixas clínica e não-clínica; e teste de Mann Whitney para comparação da variação em função do resultado do tratamento.

\section{Resultados}

A Tabela 1 mostra os escores médios obtidos para a amostra como um todo, antes e após o tratamento.

Houve redução dos escores médios nas três escalas globais (DI, DE e DT), o que significa que as mães apontaram menos problemas de comportamento após o tratamento. Essa redução foi significativa para os três escores médios (DI, DE e DT), sendo que a mais expressiva foi a redução no escore médio da escala total (DT).

A Tabela 2 apresenta as variações nos escores das escalas globais, comparando-se os participantes que alcançaram e não alcançaram sucesso no tratamento da enurese, ou seja, obtiveram pelo menos 14 noites consecutivas sem episódios de enurese durante o período de tratamento.

Verifica-se que a variação nos escores de problemas de comportamento que ocorrem na amostra como um todo, observada na Tabela 1, não guarda relação com o resultado do tratamento. Os escores médios são reduzidos de forma similar entre os que obtêm e não obtêm sucesso no controle da enurese noturna.

A Tabela 3 aponta o número de participantes com escores situados nas faixas clínica e não clínica dos escores médios de cada escala global antes e após o tratamento.

Os valores $p$ assinalados com asterisco indicam variações significativas no número de participantes com escores situados em cada faixa. No DI, por exemplo, houve uma redução de participantes com escores na faixa clínica, que passaram de 34 para 26. Já os participantes com escores não clínicos subiram de 27 para 35 . Ambas as mudanças não foram estatisticamente significativas. Na escala DE, 25 participantes tinham escores clínicos antes do tratamento, passando a 13 após a intervenção. Os escores não clínicos passaram de 36 a 48, denotando uma variação significativa. Também foi significativa a variação na escala DT, em que os 36 participantes com escores clínicos inicialmente se reduziram a 23, enquanto o número de participantes com escores não clínicos subiu de 25 para 38 .

Tabela 1. Variação dos escores médios das escalas globais do CBCL antes e após o tratamento.

\begin{tabular}{ccccc}
\hline $\begin{array}{c}\text { Antes do } \\
\text { tratamento } \\
M(d p)\end{array}$ & $\begin{array}{c}\text { Depois do } \\
\text { tratamento } \\
M(d p)\end{array}$ & Variação & $p$ \\
\hline$D I$ & $60,1(9,91)$ & $55,5(10,32)$ & $-6,76 \%$ & 0,001 \\
$D E$ & $57,8(9,43)$ & $54,2(8,86)$ & $-5,11 \%$ & 0,006 \\
$D T$ & $61,2(8,94)$ & $55,8(9,79)$ & $-8,16 \%$ & $<0,001$ \\
\hline
\end{tabular}

\section{Discussão}

Foi encontrada uma diminuição significativa nos escores dos CBCL, sugerindo que as mães apontaram menos problemas de comportamento no questionário preenchido ao final do tratamento. A diminuição chegou a resultar em mudanças de faixa clínica para não-clínica, o que confirma os dados encontrados por Hirasing e cols. (2002).

A diminuição ocorreu independentemente de ter sido obtido sucesso no tratamento, corroborando os resultados descritos por Longstaffe e cols. (2000) e opondo-se aos de Hirasing e cols. (2000). Não se pode, portanto, falar em melhora de outros problemas de comportamento em decorrência do término da enurese.

É possível que o atendimento semanal oferecido tenha tido efeitos positivos sobre o comportamento em geral de algumas crianças e adolescentes, ainda que o foco desse atendimento fosse a motivação e adesão ao tratamento para enurese. Justamente, Longstaffe e cols. (2000) sugerem que a atenção e suporte oferecidos no tratamento podem ser benéficos para todos.

Outra possibilidade é de que o atendimento tenha alterado a forma como algumas mães observam o comportamento de seus filhos, uma vez que também foram realizados atendimentos de orientação aos pais. As mudanças nos escores póstratamento poderiam ser reflexos de mudanças na identificação de comportamentos-problema. Para se investigar melhor essa hipótese, poderiam ser incluídos outros informantes que não tenham participado do atendimento.

Uma variável não controlada que poderia ter efeito sobre o preenchimento do primeiro questionário é o contexto de busca de atendimento. Como o tratamento é gratuito e geralmente há fila de espera, as mães poderiam imaginar que, apontando mais problemas de comportamento, irão ser atendidas mais rápido, ou irão garantir algum outro ganho relativo ao atendimento, o que não é verdadeiro.

Este trabalho poderia ter encontrado mais respostas e, consequentemente, oferecer mais segurança nas suas conclusões se houvesse um grupo-controle. Há, contudo, uma questão prática que dificulta a implementação dos grupos-controle, que é a espera prolongada. Para reunir um número suficiente de participantes a fim de realizar uma seleção randômica entre os que recebem ou não tratamento, seria necessário criar uma fila de espera muito extensa que, segundo a experiência dos pesquisadores, resultaria em um alto índice de desistência.

Outro aspecto que pode ter impedido a formulação de conclusões mais seguras foi a exclusão das famílias desistentes.

Tabela 2. Variação dos escores médios do CBCL de acordo com o resultado do tratamento.

\begin{tabular}{cccc}
\hline & $\begin{array}{c}\text { Sucesso } \\
M(d p)\end{array}$ & $\begin{array}{c}\text { Insucesso } \\
M(d p)\end{array}$ & $p$ \\
\hline DI & $-6,38(8,97)$ & $-8,96(8,17)$ & 0,959 \\
DE & $-4,29(7,10)$ & $-9,8(12,95)$ & 0,548 \\
DT & $-7,50(7,81)$ & $-11,9(12,35)$ & 0,784 \\
\hline
\end{tabular}


Tabela 3. Número (n) de participantes com escores clínicos e não-clínicos antes e após o tratamento.

\begin{tabular}{|c|c|c|c|c|c|}
\hline & \multirow[t]{2}{*}{ Variável } & \multicolumn{2}{|c|}{ Pós-tratamento } & \multirow[b]{2}{*}{ Total \% (n) } & \multirow{2}{*}{$\begin{array}{c}\text { Comparação entre Pré e Pós } \\
\text { (Teste de Proporção) } \\
p\end{array}$} \\
\hline & & $\begin{array}{c}\text { Não Clínico \% } \\
\text { (n) }\end{array}$ & Clínico \% (n) & & \\
\hline \multicolumn{6}{|l|}{ DI } \\
\hline \multirow{3}{*}{ 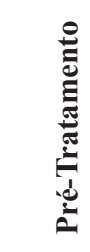 } & Não Clínico \% (n) & $36,1(22)$ & $8,2(5)^{*}$ & $44,3(27)$ & 0,096 \\
\hline & Clínico \% (n) & $21,3(13)^{*}$ & $34,4(21)$ & $55,7(34)$ & \\
\hline & Total \% (n) & $57,4(35)$ & $42,6(26)$ & $100,0(61)$ & \\
\hline \multicolumn{6}{|l|}{ DE } \\
\hline \multirow{3}{*}{ 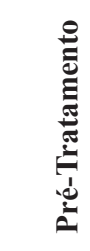 } & Não Clínico \% (n) & $55,7(34)$ & $3,3(2)^{*}$ & $59,0(36)$ & $0,004 * *$ \\
\hline & Clínico \% (n) & $23,0(14)^{*}$ & $18,0(11)$ & $41,0(25)$ & \\
\hline & Total \% (n) & $78,7(48)$ & $21,3(13)$ & $100,0(61)$ & \\
\hline \multicolumn{6}{|l|}{ DT } \\
\hline \multirow{3}{*}{ 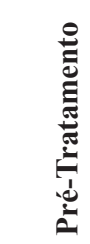 } & Não Clínico \% (n) & $36,1(22)$ & $4,9(3)^{*}$ & $41,0(25)$ & $0,004 * *$ \\
\hline & Clínico \% (n) & $26,2(16)^{*}$ & $32,8(20)$ & $59,0(36)$ & \\
\hline & Total \% (n) & $62,3(38)$ & $37,7(23)$ & $100,0(61)$ & \\
\hline
\end{tabular}

* Mudança de faixa

** Alteração significativa

A exclusão deveu-se a um fato prático, que consistiu na dificuldade em encontrar tais famílias e conseguir sua cooperação no preenchimento de questionários de finalização. Há um esforço por parte dos pesquisadores para entender os motivos da desistência e de tentar alcançar um número maior de famílias que nos possibilitem identificar mais variáveis que interferem no resultado do tratamento e nas mudanças observadas nos participantes (Sousa, Silvares \& Marturano, no prelo).

Os resultados do presente estudo tornam questionável a afirmação de Mellon e Houts (1995) de que problemas de comportamento devem ser resolvidos antes do tratamento com alarme para a enurese. Isso é importante principalmente dentro de uma realidade em que nem sempre os pais têm condições de buscar um atendimento psicológico voltado a esses problemas antes de iniciar o tratamento para a enurese. Optar por oferecer o tratamento a crianças com escores elevados de problemas de comportamento é uma decisão mais inclusiva para famílias que necessitam de apoio e que não deixa de ser efetiva.

Além disso, muitos pais buscam atendimento ou são encaminhados para tratamento devido ao incômodo e ansiedade resultantes da sintomatologia pediátrica, a queixa "orgânica" sendo elemento prepotente do esquema motivacional para o tratamento psicológico. A assistência orientada inicialmente para tratamento exclusivo, ou prioritário, das queixas comportamentais poderia retardar a atenção ao problema focal de escolha/preferência parental.

\section{Referências}

Achenbach, T. M. (1991). Integrative guide for the 1991 CBCL/ 4-18, YSR, and TRF Profiles. Burlington: University of Vermont.

Achenbach, T. M., \& Rescorla, L. A. (2007). Multicultural understanding of child and adolescent psychopathology. New York: Guilford Press.

Arantes, M. C. (2007). Problemas de comportamento e resultados do tratamento com alarme para enurese primária. Dissertação de Mestrado, Universidade de São Paulo, São Paulo.

Baeyens, D., Roeyers, H., Vande Walle, J., \& Hoebeke, P. (2005). Behavioural problems and attention-deficit hyperactivity disorder in children with enuresis: A literature review. European Journal of Pediatrics, 164, 665-672.

Bordin, I. A. S., Mari, J. J., \& Caeiro, M. F. (1995). Validação da versão brasileira do Child Behavior Checklist (CBCL) - Inventário de Comportamentos da Infância e da Adolescência: dados preliminares. Revista ABP-APAL / Associação Brasileira de Psiquiatria Asociación Psiquiatrica de la America Latina, 17, 55-66. 
Costa, N. J. D. (2005). A enurese noturna na adolescência e a intervenção comportamental em grupo $x$ individual com uso de aparelho nacional de alarme. Dissertação de Mestrado, Universidade de São Paulo, São Paulo.

Devlin, J., \& O'Cathain, C. (1990). Predicting treatment outcome in nocturnal enuresis. Archives of Disease in Childhood, 65, 1158-1161.

Ehrlick, A. L., Mellon, M. W., Whiteside, S. P., Witts, B., \& Choma, K. (2005). The relationship between child behavior and outcome in enuresis conditioning treatment. Journal of Developmental \& Behavioral Pediatrics, 26, 460.

Geffken, G., Johnson, S. B., \& Walker, D. (1986). Behavioral interventions for childhood nocturnal enuresis: The differential effect of bladder capacity on treatment progress and outcome. Health Psychology, 5, 261-272.

Hirasing, R. A., Van Leerdan F. J. M., Bolk-Bennik, L. F., \& Koot, H. M. (2002). Effect of dry bed training on behavioural problems in enuretic children. Acta Paediatrica, 91, 960-964.

Longstaffe, S., Moffatt, M., \& Whalen, J. (2000). Behavioral and self-concept changes after six months of enuresis treatment: A randomized, controlled trial. Pediatrics, 105, 935-940.

Mellon, M. W., \& Houts, A. C. (1995). Eliminations disorders. Em R. T. Ammerman \& M. Hersen (Orgs.). Handbook of child behavior therapy in the psychiatric setting (pp.341-366). New York: Wiley.

Moffat, M. E. K., \& Cheang, M. (1995). Predicting treatment outcome with conditioning alarms. Scandinavian Journal of Urology and Nephrology, 173, 119-122.

Pereira, R. F. (2006). A enurese noturna na infância e na adolescência: intervenção em grupo e individual com uso de aparelho nacional de alarme. Dissertação de Mestrado, Universidade de São Paulo, São Paulo.

Sacks, S., \& De Leon, G. (1978). Training the disturbed enuretic. Behaviour Research and Therapy, 16, 296-299.
Santos, E. O. L., \& Silvares, E. F. M. (2006). Crianças enuréticas e crianças encaminhadas para clínicas-escola: um estudo comparativo da percepção de seus pais. Psicologia: Reflexão $e$ Crítica, 19, 277-282.

Silva, R. P. (2004). Enurese noturna monossintomática: intervenção comportamental em grupos de pais e em grupos de crianças com aparelho nacional de alarme. Dissertação de Mestrado, Universidade de São Paulo, São Paulo.

Silvares, E. F. M. (2002). Família, enurese e intervenção clínica comportamental. Em H. J. Guilhardi, M. B. B. P. Madi, P. P. Queiroz \& M. C. Scoz (Eds.), Sobre comportamento e cognição: Contribuições para a construção da teoria do conhecimento - Vol. 10 (pp. 79-90). São Paulo: ESETec.

Sousa, C. R. B., Silvares, E. F. M., \& Marturano, E. M. (no prelo). Dropping out of therapy for enuresis: Evaluation of reported reasons and the effect of socioeconomic status. Journal of Consulting Psychology.

Van Hoecke, E., Baeyens, D., Vande Walle, J., Hoebeke, P., \& Roeyers, H. (2003). Socioeconomic status as a common factor underlying the association between enuresis and psychopathology. Journal of Developmental and Behavioral Pediatrics, 24, 109114.

Wagner, W. G., \& Johnson, J. B. (1988). Childhood nocturnal enuresis: The prediction of premature withdrawal from behavioral conditioning. Journal of Abnormal Child Psychology, 16, 687-692.

Recebido em 05.07.07

Primeira decisão editorial em 31.07.08

Versão final em 18.08 .08

Aceito em 10.09.08 